\title{
THE SPECTRAL THEORY OF DISTRIBUTIVE CONTINUOUS LATTICES
}

BY

KARL H. HOFMANN AND JIMMIE D. LAWSON

\begin{abstract}
In this paper various properties of the spectrum (i.e. the set of prime elements endowed with the hull-kernel topology) of a distributive continuous lattice are developed. It is shown that the spectrum is always a locally quasicompact sober space and conversely that the lattice of open sets of a locally quasicompact sober space is a continuous lattice. Algebraic lattices are a special subclass of continuous lattices and the special properties of their spectra are treated. The concept of the patch topology is extended from algebraic lattices to continuous lattices, and necessary and sufficient conditions for its compactness are given.
\end{abstract}

The spectral theory of lattices serves the purpose of representing a lattice $L$ as a lattice of open sets of a topological space $X$. The spectral theory of rings and algebras practically reduces to this situation in view of the fact that for the most part one considers the lattice of ring (or algebra) ideals and then develops the spectral theory of that lattice. (The occasional complications due to the fact that ideal products are not intersections have been dealt with elsewhere, e.g. [4].)

The lattice of all ring (or algebra) ideals forms a particular kind of continuous lattice, namely an algebraic lattice. It should be the case, however, that more general continuous lattices arise in the study of certain objects endowed with both an algebraic and a topological structure. Indeed the first author has shown in a seminar report using the concept of Pedersen's ideal that the closed ideals of a $C^{*}$-algebra always form a distributive continuous lattice with respect to intersection. How widely continuous lattices occur in such contexts is, at this point, a largely uncharted sea.

We show that the spectrum of a distributive continuous lattice is a locally quasicompact sober space (see 2.6 for the definition of sobriety). This implies, e.g., that the space of closed two sided prime ideals of a $C^{*}$-algebra is locally quasicompact in the hull-kernel topology. (This is usually proved for primitive ideals by different methods.)

On the other hand, the question of what topological consequences follow

Received by the editors November 4, 1977.

AMS (MOS) subject classifications (1970). Primary 54H10; Secondary 22A30, 06A35.

Key words and phrases. Continuous lattice, spectrum, hull-kernel topology, locally quasicompact, sober, algebraic lattice, patch topology, prime. 
for a space $X$ from the lattice theoretical assumption that the lattice $O(X)$ of open sets is a continuous lattice has received a good deal of attention. In different terms, Brian Day and Max Kelly have observed (1970) that for Hausdorff $X$ the local compactness of $X$ is necessary and sufficient [1], (see also Isbell [9]). We show that if $X$ is sober, then $O(X)$ is a continuous lattice iff $X$ is locally quasicompact.

Our main device is the use of the hitherto neglected topology on a CL-object $L$ which is generated by the sets $I(x)=L \backslash \uparrow x$. The join of this topology and the Scott topology is the CL-topology, and it induces on the set of primes precisely the hull-kernel topology.

In studying the spectrum of arithmetic lattices (such as e.g., the lattice of ideals in a commutative ring) the patch topology plays an important role [3], [1]; indeed for commutative rings this topology makes the set of prime ideals into a Boolean space. We generalize the concept of the patch topology to the spectrum of a continuous distributive lattice and derive necessary and sufficient conditions for its compactness.

We gratefully acknowledge contributions from various members of the Seminar on Continuity in Semilattices, notably K. Keimel, M. Mislove, and O. Wyler. The latter part of $\$ \S 6$ and 8 draws from a seminar report of Keimel and Mislove, and the latter part of $\$ 3$ from one of Wyler.

The authors are also grateful for support received from NSF.

1. Basic concepts. We give here a brief review of the necessary basics concerning continuous lattices for the uninitiated reader. On every set $L$ with a partial order $\leqslant$ one may introduce a new relation $\ll$ as follows: $x \ll y$ if and only if for all up-directed sets $D$ the relation $y \leqslant \sup D$ implies the existence of a $d \in D$ with $x \leqslant d$. (In a complete lattice $L, x \ll y$ iff whenever $y \leqslant \sup A$, there exists a finite subset $F \subset A$ with $x \leqslant \sup F$.) This relation, sometimes called the relation of being "way below", is readily seen to be transitive, and if $L$ has a least element 0 , then $0 \ll x$ for all $x$. The relation $x \ll y$ always implies $x \leqslant y$; the converse fails in general.

Definition. A complete lattice $L$ is said to be a continuous lattice if $x=\sup \{s \in L: s \ll x\}$ for all $x \in L$. The following facts are implicit in [7].

Proposition. For a lattice $L$ the following conditions are equivalent:

(1) There is a compact Hausdorff topology on $L$ such that $L$ becomes a topological semilattice with a basis of subsemilattices relative to the multiplication $(x, y) \rightarrow x y=\min \{x, y\}$.

(2) $L$ is a continuous lattice.

If these conditions are satisfied, then the topology is unique and is generated by the sets $\{s \in L: x \leqslant s\}$ and $\{s \in L: x \ll s\}, x \in L$; it is called the CL-topology. 
A function $f: L \rightarrow L^{\prime}$ between continuous lattices is a continuous semilattice morphism (relative to the CL-topologies) iff (i) inf $f(X)=f$ (inf $X$ ) for all $X \subseteq L$, (ii) whenever $D \subseteq L$ is up-directed, then $\sup f(D)=f(\sup D)$.

Classically, a certain subcategory $\underline{Z}$ of $C L$ is more familiar to the lattice theoretician: It is that subcategory whose objects are characterized topologically by being 0 -dimensional in the CL-topology, and lattice theoretically by being (complete) algebraic lattices; as a reminder we formulate for the record:

Definition. An element $k$ in a partially ordered set $L$ is a compact element iff $k \ll k$. The set of all compact elements of $L$ is called $K(L)$. A lattice $L$ is called algebraic iff $L$ is complete and satisfies

$$
x=\sup \{k \in L: k \leqslant x \text { and } k \in K(L)\} .
$$

The relation between topological and lattice theoretical properties of algebraic lattices was amply investigated in [6].

Let $\mathrm{CL}^{\mathrm{op}}$ denote the category of all continuous lattices whose morphisms $f$ : $L \rightarrow L^{\prime}$ satisfy (i) $\sup f(A)=f(\sup A)$ for all $A \subset L$ and (ii) $x \ll x^{\prime}$ implies $f(x) \ll f\left(x^{\prime}\right)$ for all $x, x^{\prime} \in L$. It is shown in [7] that this category is dual to $\mathrm{CL}$ under the Galois connection of order-preserving mappings, i.e. a function $f: L \rightarrow M$ is a CL-morphism iff its right adjoint $g: M \rightarrow L$ defined by $g(m)=\inf \{x: f(x) \geqslant m\}$ is a $\mathrm{CL}^{\mathrm{op}}$-morphism.

For a partially ordered set $S$, the lower set of a subset $X$ is denoted by

$$
\downarrow X=\{s \in S: s \leqslant x \text { for some } x \in X\} .
$$

$\uparrow X$ is defined dually. We denote $\downarrow\{x\}$ and $\uparrow\{x\}$ by $\downarrow x$ and $\uparrow x$ respectively.

In almost all classical theorems representing complete distributive lattices as rings of sets, one uses heavily the fact that one has an abundance of prime elements. In a semilattice $S$ an element $p$ is prime if $a b \leqslant p$ implies $a \leqslant p$ or $b \leqslant p$. Let PRIME $S$ denote the set of prime elements. Then it has been shown in [5] that if $S$ is a distributive continuous lattice, PRIME $S$ order generates $S$, i.e., $x=\inf ($ PRIME $S \cap \uparrow x$ ) for all $x \in S \backslash\{1\}$. Hence such lattices have an abundance of primes.

\section{The spectrum.}

DEFINITION. Let $L$ be a complete lattice and let $\Sigma \subset$ PRIME $L \backslash\{1\}$. If $X \subset L$ we write $h_{\Sigma}(X)=\uparrow X \cap \Sigma$ (and abbreviate $h_{\Sigma}(\{x\})$ by $h_{\Sigma}(x)$ ). Similarly we set $\sigma_{\Sigma}(X)=\Sigma \backslash h_{\Sigma}(X)=\Sigma \backslash \uparrow X$. We call $h_{\Sigma}(X)$ the hull of $X$ in $\Sigma$. The topology of $\Sigma$ is generated by the sets $\sigma_{\Sigma}(x)=\Sigma \backslash h_{\Sigma}(x)$ for all $x \in L$ and is called the hull-kernel topology. If $\Sigma=\operatorname{PRIME~} L \backslash\{1\}$ then $\Sigma$ equipped with the hull-kernel topology is called the spectrum of $L$ (or the prime spectrum, if confusion should ever arise), and denoted Spec $L$. We denote $\sigma_{\mathrm{Spec} L}$ simply by $\sigma$.

In general Spec $L$ may be empty; however if $L$ is a distributive continuous 
lattice, then PRIME $L$ order generates $L$ [5] and hence is substantial.

In this section we develop some of the basic properties of Spec $L$. Most of the results are not new, but are developed in a way convenient for us to utilize. The ultimate aim is to study the representation of $L$ in the lattice of open sets of Spec $L$. The reader may wish to bear in mind such analogs as the representation of Boolean lattices as the compact open subsets of a Boolean space or the Gelfand transform for commutative Banach algebras.

2.2. LEMMA. Let $L$ be a complete lattice, $\Sigma \subset \operatorname{Spec} L$.

(a) $\cap\left\{h_{\Sigma}(x): x \in X\right\}=h_{\Sigma}(\sup X)$ for all $X \subset L$.

(b) $\bigcup\left\{h_{\Sigma}(x): x \in X\right\}=h_{\Sigma}(X)=h_{\Sigma}($ inf $X)$ for all finite $X \subset L$.

(c) Every hull-kernel closed set of $\Sigma$ is of the form $h_{\Sigma}(x)$ for some $x \in L$.

(d) If $L$ is a continuous lattice endowed with the $\mathrm{CL}$-topology, then for all compact subsets $X \subset L, \cup\left\{h_{\Sigma}(x): x \in X\right\}=h_{\Sigma}(X)=h_{\Sigma}(\inf X)$.

Proof. (a) is straightforward.

(b) Clearly $\cup\left\{h_{\Sigma}(x): x \in X\right\} \subset h_{\Sigma}(\inf X)$. Conversely if $p \in h_{\Sigma}(\inf X)$, then inf $X<p$. Since $p$ is prime and $X$ is finite, $x<p$ for some $x \in X$. Hence $p \in \cup\left\{h_{\Sigma}(x): x \in X\right\}$.

(c) The family $\left\{h_{\Sigma}(x): x \in L\right\}$ is closed under arbitrary meets by (a) and under finite unions by (b). It is therefore the set of closed sets of a topology, the hull-kernel topology.

(d) Again the containment $\cup\left\{h_{\Sigma}(x): x \in X\right\} \subset h_{\Sigma}($ inf $x)$ is immediate. Conversely if $X$ is compact and inf $X \leqslant p \in \Sigma$, then by "THE LEMMA" [2], $x<p$ for some $x \in X$. Hence $h_{\Sigma}(\inf X) \subset \cup\left\{h_{\Sigma}(x): x \in X\right\}$. $\square$

REMARKS. It follows from Lemma 2.2 that the collection $\left\{h_{\Sigma}(x): x \in L\right\}$ is closed with respect to finite unions and arbitrary intersections. Since $h_{\Sigma}(0)=$ $\Sigma$ and $h_{\Sigma}(1)=\varnothing$, this collection forms all the closed sets for the hull-kernel topology on $X$. Thus $\left\{\sigma_{\Sigma}(x): x \in L\right\}$ is the collection of open sets.

If $X$ is a topological space, let $O(X)$ denote the lattice of open sets of $X$. We consider now the representation of $L$ in $O(\Sigma)$.

2.3. Proposition. Let $L$ be a complete lattice, $\Sigma \subset \operatorname{Spec} L$. Then the function $\sigma_{\Sigma}$ from $L$ to the lattice of open sets $O(\Sigma)$ which sends $x$ to $\sigma_{\Sigma}(x)$ is a surjective lattice homomorphism preserving arbitrary sups. The following conditions are equivalent:

(1) $\sigma_{\Sigma}$ is an isomorphism;

(2) $\sigma_{\Sigma}$ is injective;

(3) $\Sigma$ is order generating (i.e. $x=\inf (\uparrow x \cap \Sigma)$ for all $x \in L \backslash\{1\})$.

These conditions imply

(4) $L$ is distributive, and if $L$ is continuous and $\Sigma=\operatorname{Spec} L$, then all four conditions are equivalent. 
Proof. The first assertion follows from Lemma 2.2 and the remarks following it.

The equivalence of (1) and (2) is obvious since $\sigma_{\Sigma}$ is a surjective homomorphism. If $\Sigma$ is order generating, then $x=\inf h_{\Sigma}(x)$ for all $x \in L$. This implies $\sigma_{\Sigma}$ is injective.

Conversely if $\sigma_{\Sigma}$ is injective, then $\Sigma$ is order generating since always $\sigma_{\Sigma}(x)=\sigma_{\Sigma}(\inf (\uparrow x \cap \Sigma))$. It is well known that a complete lattice in which the primes order generate is distributive and the converse is true in continuous lattices [5, 3.1].

This proposition has the important consequence that all distributive continuous lattices can be represented in the form $O(X)$.

If $\sigma: L \rightarrow M$ and $\tau: M \rightarrow L$, then $\tau$ is a left adjoint for $\sigma$ (and $\sigma$ is a right adjoint for $\tau$ ) if for $x \in L, y \in M$ we have $\sigma(x) \leqslant y$ iff $x \leqslant \tau(y)$. If $\sigma$ : $L \rightarrow M$ is a function between complete lattices which preserves arbitrary sups, then it has a unique left adjoint $\tau: M \rightarrow L$ which preserves arbitrary infs and is defined by $\tau(y)=\sup \{x \in L: \sigma(x)<y\}$. (See the early part of [7] for an extended discussion of such matters.)

2.4. Proposition. Let $\sigma: L \rightarrow M$ be a lattice homomorphism preserving arbitrary sups and 1. If $\tau: M \rightarrow L$ is the left adjoint for $\sigma$, then $\tau(\operatorname{Spec} M) \subset$ Spec $L$ and $\tau$ restricted to Spec $M$ is continuous for the hull-kernel topologies.

Proof. Let $p \in \operatorname{Spec} M$. Since $\tau(p)=\sup \{x: \sigma(x)<p\}$ and $\sigma(1)=1$, $\tau(p) \neq 1$. Let $s t \leqslant \tau(p)$. Then $s t \leqslant \tau(p)$ iff $\sigma(s t) \leqslant p$ iff $\sigma(s) \sigma(t) \leqslant p$ iff $\sigma(s) \leqslant p$ or $\sigma(t) \leqslant p$ iff $s \leqslant \tau(p)$ or $t \leqslant \tau(p)$. Hence $\tau(p)$ is prime. That the restriction of $\tau$ is continuous follows from the fact $\tau$ preserves arbitrary infs. We omit the details.

Proposition 2.4 shows that Spec may be viewed as a contravariant functor from the category of complete lattices and lattice homomorphisms preserving arbitrary sups to the category of topological spaces and continuous functions.

Notation. For $\Sigma \subset \operatorname{Spec} L$, let $L_{\Sigma}$ denote the inf-complete subsemilattice generated by $\Sigma \cup\{1\}$, i.e.,

$$
L_{\Sigma}=\{\inf A: A \subset \Sigma\}
$$

(where inf $\varnothing=1$ ). Note that $L_{\Sigma}$ is order generated by prime elements, and is hence distributive.

2.5. Proposition. The function $\sigma_{\Sigma}: L \rightarrow O(\Sigma)$ has a left adjoint $\tau_{\Sigma}: O(\Sigma) \rightarrow$ $L$ given by $\tau_{\Sigma}(U)=\inf (\Sigma \backslash U)$. The function $\tau_{\Sigma}$ is an injection, preserves arbitrary infs, and has image $L_{\Sigma}$. The restriction of $\sigma_{\Sigma}$ and the corestriction of $\tau_{\Sigma}$ to $L_{\Sigma}$ are mutual inverses.

The following statements hold:

(i) $\tau_{\Sigma}(U) \in \operatorname{Spec} L$ iff $U \in \operatorname{Spec} O(\Sigma)$ iff $A=\Sigma \backslash U$ is an irreducible closed 
set (i.e. a nonempty closed set which is not the union of two proper closed subsets).

(ii) $A$ is an irreducible closed set iff $A=h(x)$ for some $x \in \operatorname{Spec} L \cap L_{\Sigma}$.

(iii) $\tau_{\Sigma}$ preserves sups of up-directed sets iff $L_{\Sigma}$ contains the sups of all its up-directed subsets.

Proof. To see that $\tau_{\Sigma}$ is indeed the left adjoint, observe that inf $(\Sigma \backslash U) \geqslant x$ iff $\Sigma \backslash U \subset \uparrow x$ iff $\sigma_{\Sigma}(x)=\Sigma \backslash \uparrow x \subset U$; thus $\tau_{\Sigma}(U) \geqslant x$ iff $U \supset \sigma_{\Sigma}(x)$, which is precisely the condition that $\tau_{\Sigma}$ be a left adjoint.

The next assertions will follow if it is shown that the restriction of $\sigma_{\Sigma}$ to $L_{\Sigma}$ is surjective and that $\tau_{\Sigma} \sigma_{\Sigma}(x)=x$ for all $x \in L_{\Sigma}$. Let $U \in O(\Sigma)$. Then $U=\sigma_{\Sigma}(y)$ for some $y \in L$. Let $x=\inf \Sigma \cap \uparrow y=\inf h(y)$. Then $x \in L_{\Sigma}$ and $h(x)=h(y)$; hence $\sigma_{\Sigma}(x)=\sigma_{\Sigma}(y)=U$. Also if $x \in L_{\Sigma}$, then $x=\inf \Sigma$ $\cap \uparrow x=\inf h(x)$. Thus $\tau_{\Sigma} \sigma_{\Sigma}(x)=\inf \left(\Sigma \backslash \sigma_{\Sigma}(x)\right)=\inf h(x)=x$.

Ad (i). If $U \in \operatorname{Spec} O(\Sigma)$, then $\tau_{\Sigma}(U) \in \operatorname{Spec} L$ by 2.4. Conversely if $\tau_{\Sigma}(U) \in \operatorname{Spec} L$, then $\tau_{\Sigma}(U) \in \operatorname{Spec} L_{\Sigma}$ (since $L_{\Sigma}=\tau_{\Sigma}(O(\Sigma))$ ) and hence $U \in \operatorname{Spec} O(\Sigma)$ since the corestriction of $\tau_{\Sigma}$ from $O(\Sigma)$ to $L_{\Sigma}$ is an isomorphism. Now $U \in \operatorname{Spec} O(\Sigma)$ iff $U$ is prime and $U \neq \Sigma$ iff $A=\Sigma \backslash U$ is coprime in the lattice of closed sets and $A \neq \varnothing$ iff $A$ is a closed irreducible set (since the lattice of closed sets is distributive).

Ad (ii). $A$ is irreducible and closed iff $U=\Sigma \backslash A \in \operatorname{Spec} O(\Sigma)$ iff $\tau_{\Sigma}(U) \in$ Spec $L \cap L_{\Sigma}$ (by (i)). Let $x=\tau_{\Sigma}(U)$. Then $x$ is the unique element in $L_{\Sigma}$ such that $A=h(x)$ (since $\sigma_{\Sigma}$ restricted to $L_{\Sigma}$ is an isomorphism). The desired result follows.

Ad (iii). Since $\tau_{\Sigma}$ is an isomorphism from $O(\Sigma)$ to $L_{\Sigma}, \tau_{\Sigma}$ preserves the sups of up-directed sets iff the sups of the images of these sets lie in $L_{\Sigma}$ iff $L_{\Sigma}$ contains the sups of all its up-directed subsets.

To this point we have begun with a complete lattice $L$ and derived a topological space $\operatorname{Spec} L$. We now wish to reverse the procedure. To each topological space $X$ we associate the complete lattice of open sets $O(X)$. If $f$ : $X \rightarrow Y$ is a continuous function, then there is induced a lattice homomorphism $O(f): O(Y) \rightarrow O(X)$ which preserves arbitrary joins and 1 defined by sending $U$ to $f^{-1}(U)$. (Compare with the remarks following 2.4.)

2.6. Definition. A space $X$ is sober if it is $T_{0}$ and every closed irreducible set has a dense point.

Note that the closure of a point is always an irreducible closed set. Hausdorff spaces are sober, while any infinite set with the cofinite topology is a nonsober $T_{1}$-space.

For every topological space $X$, the lattice of open sets $O(X)$ is a complete Brouwerian lattice (or Heyting algebra). We let $\operatorname{Spec} O(X)$ be the space of its primes in the hull-kernel topology, the set $\{\sigma(U): U \in O(X)\}$, where $\sigma(U)=$ $\{P \in \operatorname{Spec} O(X): U \not \subset P\}$. (For further information see e.g. [4], but be 
careful in comparing notation.) The importance of sober spaces is that they are precisely those spaces which can be recovered from their lattice of open sets as the following proposition specifies (see part (v)).

2.7. Proposition. Let $X$ be a topological space and define $\xi: X \rightarrow \operatorname{Spec} O(X)$ by $\xi(x)=X \backslash\{x\}^{-}$. Then $\xi$ has the following properties:

(i) For all $U \in O(X)$ we have

(a) $\xi(U)=\sigma(U) \cap \operatorname{im} \xi$ and

(b) $U=\xi^{-1}(\sigma(U))$.

(ii) $\sigma: O(X) \rightarrow O(\operatorname{Spec} O(X))$ is a lattice isomorphism with inverse $V \rightarrow$ $\xi^{-1}(V)$.

(iii) $\xi$ is continuous and open onto its image.

(iv) $\xi$ is injective iff $\xi$ is an embedding iff $X$ is $T_{0}$.

(v) $\xi$ is bijective iff $\xi$ is a homeomorphism iff $X$ is sober.

(vi) Spec $O(X)$ is sober.

Proof. (i) (a) An open set $P \in \operatorname{Spec} O(X)$ is in $\sigma(U)$ iff $U \not \subset P$; hence $X \backslash\{x\}^{-}$is in $\sigma(U) \cap \operatorname{im} \xi$ iff $U \not \subset X \backslash\{x\}^{-}$iff $x \in U$ iff $X \backslash\{x\}^{-} \in$ $\xi(U)$.

(b) An element $x \in X$ is in $\xi^{-1}(\sigma(U))$ iff $\xi(x) \in \sigma(U)$ iff $U \not \subset X \backslash\{x\}^{-}$ iff $x \in U$.

(ii) is a consequence of (i)(b) and the fact that $\sigma$ is surjective.

(iii) follows from (i)(b) and (a), respectively.

(iv) and (v) are immediate from the definitions in view of (iii).

(vi) Since $\sigma: O(X) \rightarrow O(\operatorname{Spec} O(X))$ is a lattice isomorphism by (ii), it follows that the induced $\xi^{\prime}: \operatorname{Spec} O(X) \rightarrow \operatorname{Spec}(O(\operatorname{Spec} O(X)))$ is a homeomorphism. By (v) Spec $O(X)$ is sober.

We abbreviate Spec $O(X)$ by $\check{X}$; then ${ }^{-}$is a functor from the category of $\left(T_{0}-\right)$ spaces into the category of sober spaces. In fact it is a left adjoint to the inclusion functor. Specifically:

2.8. Proposition. If $S$ is a sober space then every continuous function $f$ : $X \rightarrow S$ factors uniquely through $\xi_{X}: X \rightarrow \check{X}$.

Proof. By the naturality of $\xi$ there is a continuous diagram

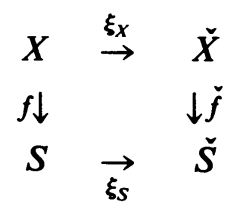

but since $S$ is sober, $\xi_{S}$ is an isomorphism by $2.7(\mathrm{v})$. Thus the desired factorization exists. If we had a relation $g \xi_{X}=\xi_{S} f$, then upon applying the functor $O$ we would derive $O(g) O\left(\xi_{X}\right)=O\left(\xi_{S} f\right)=O(\check{f}) O\left(\xi_{X}\right)$, whence 
$O(g)=O(\check{f})$ since $O\left(\xi_{X}\right)$ is an isomorphism by $2.7(\mathrm{ii})$. But then $g=\check{f}$ since $O$ is faithful on sober spaces.

2.9. Definition. We call $\check{X}$ the sobrification of $X$.

2.10. Proposition. Let $L$ be a complete lattice, $\Sigma \subset \operatorname{Spec} L$. If $\Sigma$ is order generating, then a closed subset $A$ of $\Sigma$ is irreducible iff $A=h_{\Sigma}(a)=\uparrow a \cap \Sigma$ for some $a \in \operatorname{Spec} L$, i.e. $A=\{a\}^{-} \cap \Sigma$ for some $a \in \operatorname{Spec} L$. Hence $\operatorname{Spec} L$ is sober if it is order generating.

Proof. Immediate from 2.5(ii).

3. Spectra of continuous lattices. In this section we consider specifically the spectrum of a continuous lattice and subspaces thereof. Although several of the results are set in a more general context, continuous lattices are really the motivation.

Recall that in a complete lattice $L$ a set $U$ is Scott-open if $\uparrow U=U$ and sup $D \in U$ implies $d \in U$ for some $d \in D$ if $D$ is an up-directed set (equivalently $\sup A \in U$ implies sup $F \in U$ for some finite $F \subset A$ ). If $L$ is a continuous lattice and $U=\uparrow U \subset L$, then it follows that $U$ is open in the Scott topology iff $U$ is open in the CL-topology.

Since many of the spaces dealt with in this paper are not Hausdorff, we adopt the Bourbaki convention that a space is quasicompact if every open cover has a finite subcover and compact if it is both quasicompact and Hausdorff. For a continuous lattice the Scott topology is $T_{0}$ and quasicompact and the CL-topology is compact. In the remainder of the paper a compact subset of a lattice L means compact in the CL-topology.

Let $L$ denote a complete lattice.

3.1. LEMMA. For $\Sigma \subset \operatorname{Spec} L$ and a filter $F$ of $L$ the following are equivalent:

(1) For all $x \in L, \Sigma \cap \uparrow x \subset F$ implies $x \in F$;

(2) For all $x \in L, x \notin F$ implies there exists $p \in \Sigma \backslash F$ with $x<p$;

(3) $\downarrow(\Sigma \backslash F)=L \backslash F$.

Proof. Straightforward.

3.2. Definition. A filter $F \subset L$ is called $\Sigma$-compatible if the equivalent conditions of Lemma 3.1 are satisfied by $F$, e.g., $\downarrow(\Sigma \backslash F)=L \backslash F$.

3.3. Proposition. (A) If $\Sigma=\operatorname{Spec} L$ and $L$ is distributive, then every Scott open filter is $\Sigma$-compatible.

(B) If Irr $L \backslash\{1\} \subset \Sigma$, then every Scott open principal filter is $\Sigma$-compatible (where Irr $L$ denotes the completely irreducible elements).

Proof. Let $F$ be an open filter, $x \notin F$. Let $M$ be a maximal chain containing $x$ in $L \backslash F$. Then $p=\sup M \in L \backslash F$ since $F$ is Scott open. It follows easily that $p$ is meet-irreducible and hence prime if $L$ is distributive. If 
also $F$ is principal, then $p$ is completely irreducible. (See Lemma 1.4 of [5].)

3.4. Lemma. Let $L$ be a complete lattice, $\Sigma \subset \operatorname{Spec} L$. Let $Q \subset \Sigma$. The following statements are equivalent:

(1) $Q$ is quasicompact.

(2) $\downarrow Q$ is closed in the Scott topology.

(3) $\downarrow Q \cap \Sigma$ is quasicompact.

(4) There exists a $\Sigma$-compatible Scott-open filter $F$ such that $\sigma_{\Sigma}(F)=\Sigma \backslash F$ $=\downarrow Q \cap \Sigma$.

Proof. (1) $\Leftrightarrow(2)$. A family $\left\{\sigma_{\Sigma}(a): a \in A\right\}$ of open sets in $\Sigma$ is a cover of $Q$ iff $Q \subset \cup\left\{\sigma_{\Sigma}(a): a \in A\right\}=\sigma_{\Sigma}(\sup A)$ iff $Q \backslash h(\sup A)=\varnothing$ iff $\sup A \notin$ $\downarrow Q$. Thus $Q$ has the Heine-Borel property iff for each set $A \subset L$ with $\sup A \notin \downarrow Q$, there is a finite subset $F \subset A$ with $\sup F \notin Q$. This means precisely that $L \backslash \downarrow Q$ is open in the Scott topology.

(1) $\Leftrightarrow(3)$. The set $\left\{\sigma_{\Sigma}(a): a \in A\right\}$ is an open cover of $Q$ iff it is an open cover of $\downarrow Q \cap \Sigma$. The equivalence follows.

(2) $\Rightarrow(4)$. Let $F=L \backslash \downarrow Q$. Then $F$ is Scott-open. Since $F=\cap\{L \backslash \downarrow p$ : $p \in Q\}$ and each $L \backslash \downarrow p$ is a filter as $p$ is prime, we have $F$ is a filter. Now $\sigma_{\Sigma}(F)=\Sigma \backslash F=\Sigma \backslash(L \backslash \downarrow Q)=\Sigma \cap \downarrow Q$. Finally if $x \notin F$, then $x \in \downarrow Q$. Hence there exists $p \in Q \subset \Sigma$ such that $x \leqslant p$. Since $Q \subset \Sigma \cap \downarrow Q=\Sigma \backslash F$, we have $p \notin F$. Thus $F$ is $\Sigma$-compatible.

(4) $\Rightarrow$ (2). By hypothesis $L \backslash F$ is Scott-closed. To complete the proof we show $\downarrow Q=L \backslash F$. Since $Q \subset \downarrow Q \cap \Sigma \subset L \backslash F$ and $F$ is a filter, we conclude $\downarrow Q \subset L \backslash F$. Let $x \in L \backslash F$. Since $F$ is $\Sigma$-compatible, there exists $p \in \Sigma \backslash F$ such that $x<p$. But $\Sigma \backslash F=\downarrow Q \cap \Sigma$. Thus $p \in \downarrow Q$ and hence $x \in \downarrow Q$.

3.5. Definition. A topological space $X$ is called locally quasicompact if every point has arbitrarily small quasicompact neighborhoods.

Note that in the absence of separation the existence of one quasicompact neighborhood is not sufficient to guarantee local quasicompactness.

3.6. Lemma. Let $X$ be a topological space.

(a) If $U, V \in O(X)$ and $Q$ is quasicompact with $U \subset Q \subset V$, then $U \ll V$ in $O(X)$.

(b) If $X$ is locally quasicompact, then $O(X)$ is a continuous lattice such that for $U, V \in O(X)$ the following are equivalent:

(1) $U \ll V$.

(2) There exists a quasicompact $Q \subset X$ with $U \subset Q \subset V$.

Proof. (a) Note that in $O(X)$ sups are unions. If $U$ is a collection of open sets and $V \subset \cup Q$, then $U \subset Q \subset U_{1} \cup \cdots \cup U_{n}$ since $Q$ is quasicompact for some $U_{1}, \ldots, U_{n} \in \mathcal{Q}$. Thus $U \ll V$.

(b) Let $V \in O(X), x \in V$. There exists a quasicompact neighborhood $Q$ of 
$x$ such that $Q \subset V$. Then $x \in \operatorname{int}(Q) \subset V$ and $\operatorname{int}(Q) \ll V$ by part (a). Thus $V=U\{U \in O(X): U \ll V\}$. Hence $O(X)$ is a continuous lattice.

Again (2) implies (1) by part (a). Conversely assume $U \ll V$. For each $x \in V$, pick $Q_{x}$ quasicompact such that $Q_{x} \subset V$. Then $\cup$ int $\left.Q_{x}: x \in V\right\}$ covers $V$; hence there exist $Q_{1}, \ldots, Q_{n}$ such that $U \subset$ int $Q_{1} \cup \cdots \cup$ int $Q_{n}$. Thus if $Q=Q_{1} \cup \cdots \cup Q_{n}$, then $Q$ is a quasicompact set such that $U \subset Q \subset V$.

3.7. Proposition. Let $L$ be a complete lattice, $a, b \in L$, and $\Sigma \subset \operatorname{Spec} L$. The following statements are equivalent:

(1) There is a quasicompact set $Q$ in $\Sigma$ with $\sigma_{\Sigma}(a) \subset Q \subset \sigma_{\Sigma}(b)$;

(2) There is a $\Sigma$-compatible open filter $F$ in $L$ with $b \in F, F \cap \Sigma \subset h_{\Sigma}(a)$.

Thus $\Sigma$ is locally quasicompact iff for $p \in \Sigma$ and $x \leqslant p$, there exists $a$ $\Sigma$-compatible open filter $F$ such that $x \in F$ and $\inf (F \cap \Sigma)<p$.

Proof. (1) $\Rightarrow(2)$. As in the proof of 3.4, $F=L \backslash \downarrow Q$ is an open $\Sigma$-compatible filter. The relations $b \in F$ and $F \cap \Sigma \subset h_{\Sigma}(a)$ are straightforward.

(2) $\Rightarrow(1)$. By $3.4 Q=\Sigma \backslash F$ is quasicompact. Also $\sigma_{\Sigma}(a)=\Sigma \backslash h_{\Sigma}(a) \subset \Sigma \backslash$ $F=Q$ and $Q=\Sigma \backslash F=\sigma_{\Sigma}(F) \subset \sigma_{\Sigma}(\uparrow b)=\sigma_{\Sigma}(b)$.

Now assume $\Sigma$ is locally quasicompact, $p \in \Sigma$ and $x \nless p$. Then $p \in \sigma_{\Sigma}(x)$. Thus there exists $y \in L$ and a quasicompact set $Q \subset \Sigma$ such that $p \in \sigma_{\Sigma}(y)$ $\subset Q \subset \sigma_{\Sigma}(x)$. By what we have just shown there exists a $\Sigma$-compatible open filter $F$ in $L$ with $x \in F, h_{\Sigma}(y) \supset F \cap \Sigma$. Hence $\inf (F \cap \Sigma) \geqslant y$. Since $p \in \sigma_{\Sigma}(y), \inf (F \cap \Sigma) \nless p$.

Conversely let $p \in \sigma_{\Sigma}(x)$. Then $x \nless p$; hence there exists a $\Sigma$-compatible open filter $F$ such that $x \in F$ and $y=\inf (F \cap \Sigma) \nless p$. Now $F \cap \Sigma \subset h_{\Sigma}(y)$, so by the first part of the proposition we have the existence of a quasicompact set $Q$ such that $p \in \sigma_{\Sigma}(y) \subset Q \subset \sigma_{\Sigma}(x)$.

We turn our attention now to the case that $L_{\Sigma}$, the inf-complete semilattice generated by $\Sigma$, is actually a continuous lattice. Note that the hull-kernel topologies on $\Sigma$ defined by $L$ and $L_{\Sigma}$ resp. agree since $h_{\Sigma}(x)=h_{\Sigma}(\inf (\Sigma \cap$ $\uparrow x)$ ). Hence we may pass back and forth between them.

3.8. TheOREM. Let $L$ be a complete lattice, $\Sigma \subset \operatorname{Spec} L$. The following statements are equivalent:

(1) $L_{\Sigma}$ is a continuous lattice;

(2) $O(\Sigma)$ is a continuous lattice;

(3) [3'] Spec $L_{\Sigma}=\operatorname{Spec} L \cap L_{\Sigma}$ is a locally quasicompact [sober] space;

(4) $O\left(\operatorname{Spec} L_{\Sigma}\right)$ is a continuous lattice.

Furthermore $\Sigma$ is locally quasicompact iff the above equivalent conditions hold and whenever $x \ll y$ in $L_{\Sigma}$, then there exists in $L_{\Sigma}$ a $\Sigma$-compatible open filter $F$ in $L_{\Sigma}$ with $y \in F \subset \uparrow x$. 
Proof. The equivalence of (1) and (2) follows from the fact that $\sigma_{\Sigma}$ restricted to $L_{\Sigma}$ is an isomorphism by 2.5 .

In the next few paragraphs we work entirely in the lattice $L_{\Sigma}$.

The implication (3) $\Leftrightarrow(4)$ and the fact that if $\Sigma$ is locally quasicompact, then (2) follows are both consequences of 3.6(b).

Let us assume $\Sigma$ is locally quasicompact and show one implication in the last paragraph. Let $x \ll y$ in $L_{\Sigma}$. Let $U=\{w: x \ll w\}$ and let $K=L_{\Sigma} \backslash U$. Since $U$ is open in the CL-topology, $K$ is compact. For each $t \in K, y \nless t$ (otherwise $t \in U$ ). Since $\Sigma$ order generates $L_{\Sigma}$, there exists $p \in \Sigma$ such that $t \leqslant p$ but $y \nless p$. By 3.7 there exists a $\Sigma$-compatible open filter $F_{t}$ such that $y \in F_{t}$ and $\inf \left(F_{t} \cap \Sigma\right) \nless p$. But again since $\Sigma$ order generates $L_{\Sigma}, \inf \left(F_{t} \cap\right.$ $\Sigma)=\inf F_{t}=z_{t}$. Since $t \nless p, z_{t} \leqslant t$. Thus for each $t \in K, L_{\Sigma} \backslash \uparrow z_{t}$ is a CL-open set around $t$. Since $K$ is compact, there exists $z_{1}, \ldots, z_{n}$ such that $\cap{ }_{i=1}^{n} \uparrow z_{i} \subset U$. Then if $F=\bigcap_{i=1}^{n} F_{z_{i}}, F$ is a $\Sigma$-compatible open filter and $y \in F \subset \uparrow x$.

Let us assume $L_{\Sigma}$ is a continuous lattice and prove the converse. Let $p \in \Sigma$ and $x \nless p$. Since $L_{\Sigma}$ is a continuous lattice there exists $w \ll x$ such that $w \nless p$. By hypothesis there exists a $\Sigma$-compatible open filter $F$ such that $x \in F \subset \uparrow w$. Hence $w \leqslant \inf (F \cap \Sigma)$ and so $\inf (F \cap \Sigma) \nless p$. Again by 3.7 applied to $L_{\Sigma}, \Sigma$ is locally quasicompact.

We now complete the remaining implications.

(1) $\Rightarrow\left(3^{\prime}\right)$. Let $p \in \operatorname{Spec} L_{\Sigma}$. Since $\sigma_{\Sigma}$ restricted to $L_{\Sigma}$ is an isomorphism, $\sigma_{\Sigma}(p) \in \operatorname{Spec} O(\Sigma)$. Thus $\tau_{\Sigma} \sigma_{\Sigma}(p) \in \operatorname{Spec} L$ by 2.5(i). But again by $2.5 \tau_{\Sigma}$ is the inverse for the restriction of $\sigma_{\Sigma}$ to $L_{\Sigma}$; thus $\tau_{\Sigma} \sigma_{\Sigma}(p)=p$. Hence Spec $L_{\Sigma}$ $\subset$ Spec $L \cap L_{\Sigma}$. The other inclusion is immediate.

Let $x \ll y$ in $L_{\Sigma}$. By basic properties of continuous lattices there exists an open filter $F$ with $y \in F \subset \uparrow x$. By 3.3(A) $F$ is compatible for Spec $L_{\Sigma}$ (note that $L_{\Sigma}$ is distributive since it is order generated by primes). Thus it follows from the last paragraph of the theorem that $\operatorname{Spec} L_{\Sigma}$ is locally quasicompact.

Since $\Sigma$ order generates $L_{\Sigma}$ by $2.3 L_{\Sigma} \rightarrow O\left(\operatorname{Spec} L_{\Sigma}\right)$ is an isomorphism. Hence $\operatorname{Spec}\left(L_{\Sigma}\right) \rightarrow \operatorname{Spec}\left(O\left(\operatorname{Spec} L_{\Sigma}\right)\right)$ is a homeomorphism. Since by $2.7(\mathrm{vi})$ the latter is a sober space, so is $\operatorname{Spec}\left(L_{\Sigma}\right)$.

$\left(3^{\prime}\right) \Rightarrow(3)$. Immediate.

$(4) \Rightarrow(1)$. Since $\Sigma$ order generates $L_{\Sigma}$, so does $\operatorname{Spec} L_{\Sigma}$. Hence by 2.3 $L_{\Sigma} \rightarrow O\left(\operatorname{Spec} L_{\Sigma}\right)$ is an isomorphism and the result follows.

Finally we specialize to the case that $L$ is a continuous lattice and $L_{\Sigma}$ is a CL-subobject of $L$, i.e. a compact subsemilattice.

3.9. Proposition. Let $L$ be a continuous lattice, $\Sigma \subset \operatorname{Spec} L$. The following are equivalent:

(1) $L_{\Sigma}$ is compact, i.e., a CL-subobject;

(2) $L_{\Sigma}$ is closed under sups of up-directed sets; 
(3) $\sigma_{\Sigma}: L \rightarrow O(\Sigma)$ is a $\mathrm{CL}^{\mathrm{op}}$-morphism.

(4) $\tau_{\Sigma}: O(\Sigma) \rightarrow L$ is a CL-morphism.

Proof. The results of $\$ 3$ of [5] imply the equivalence of (1) and (2) (see Proposition 3.8 there for more details about this situation).

The equivalence of (3) and (4) is known from [7].

The equivalence of (2) and (4) follows from 2.5(iii).

We consider now conditions under which $\Sigma$ is locally quasicompact for this case. The equivalence of (1) and (3) in the following result was first established by $O$. Wyler (unpublished seminar report).

3.10. Proposition. Let $L$ be a continuous lattice, $\Sigma \subset \operatorname{Spec} L$. The following conditions are equivalent:

(1) $\Sigma$ is locally quasicompact, and $\sigma_{\Sigma}: L \rightarrow O(\Sigma)$ is a $\mathrm{CL}^{\mathrm{op}}-$ map.

(2) Whenever $x \ll y$ in L, there exists an open filter $F$ with $y \in F \subset \uparrow x$ such that $\Sigma \backslash F$ is quasicompact. If $\Sigma=\operatorname{Spec} L$, then (1) and (2) are equivalent to:

(3) For every open filter $F$ of $L$ the set $(\operatorname{Spec} L) \backslash F$ is quasicompact.

Proof. (1) $\Rightarrow(2)$. Let $x \ll y$ in $L$. Since $L$ is continuous, there exists $z$ with $x \ll z \ll y$. Since $\sigma_{\Sigma}$ is a $\mathrm{CL}^{\mathrm{op}}$-mapping, $\sigma_{\Sigma}(z) \ll \sigma_{\Sigma}(y)$ in $O(\Sigma)$. Since $\Sigma$ is locally quasicompact, by $3.6(\mathrm{~b})$ there exists a quasicompact set $Q$ such that $\sigma_{\Sigma}(z) \subset Q \subset \sigma_{\Sigma}(y)$. By 3.7 there exists a $\Sigma$-compatible open filter $F_{1}$ in $L$ with $y \in F_{1}$ and $\left(F_{1} \cap \Sigma\right) \subset \uparrow z$. By basic properties of continuous lattices there exists an open filter $F_{2}$ such that $z \in F_{2} \subset \uparrow x$. Then if $F=F_{1} \cap F_{2}, F$ is an open filter, $F \subset \uparrow x, y \in F$, and $F \cap \Sigma=F_{1} \cap \Sigma$ (since $F_{1} \cap \Sigma \subset \uparrow z$ and $\uparrow z \subset F_{2}$ ). Since $F_{1}$ is $\Sigma$-compatible and open, by 3.4(B) $\Sigma \backslash F=\Sigma \backslash F_{1}$ is quasicompact in $\Sigma$.

$(2) \Rightarrow(1)$. Let $p \in \sigma_{\Sigma}(a)$. Then $a \nless p$. Hence there exists $b \ll a$ such that $b \nless p$. By hypothesis there exists an open filter $F$ such that $a \in F \subset \uparrow b$ and $\Sigma \backslash F$ is quasicompact. Thus we have $p \in \sigma_{\Sigma}(b) \subset \sigma_{\Sigma}(F)=\Sigma \backslash F \subset \sigma_{\Sigma}(a)$. So $\Sigma$ is locally quasicompact.

Let $x \ll y$ and again pick an open filter $F$ such that $y \in F \subset \uparrow x$ and $\Sigma \backslash F$ is quasicompact. Then $\sigma_{\Sigma}(x) \subset \sigma_{\Sigma}(F)=\Sigma \backslash F \subset \sigma_{\Sigma}(y)$. By 3.6(a) we have $\sigma_{\Sigma}(x) \ll \sigma_{\Sigma}(y)$. Thus $\sigma_{\Sigma}$ is a $\mathrm{CL}^{\mathrm{op}}$-morphism.

$(3) \Rightarrow(2)$. This follows from the basic property of continuous lattices that $x \ll y$ implies the existence of an open filter $F$ with $y \in F \subset \uparrow x$.

$(1) \Rightarrow(3)$. Let $F$ be any open filter in $L$. Then $L_{\Sigma} \cap F$ is an open filter in $L_{\Sigma}$. By $3.9 L_{\Sigma}$ is a CL-subobject of $L$, and $L_{\Sigma}$ is distributive since it is generated by primes. Since $\Sigma=\operatorname{Spec} L$, by 3.3(A) we have $L_{\Sigma} \cap F$ is $\Sigma$-compatible in $L_{\Sigma}$. Applying 3.4 to $L_{\Sigma}$ we have $\Sigma \backslash F$ is quasicompact.

We close this section by considering two cases in which $L_{\Sigma}$ is a CL-subobject of $L$ (and hence allow the application of 3.8, 3.9, and 3.10). 
3.11. Proposition. Let $L$ be a continuous lattice, $\Sigma \subset \operatorname{Spec} L$. If $\Sigma \cup\{1\}$ is compact in $L$, then $L_{\Sigma}$ is a compact CL-subobject of $L$, and $\operatorname{Spec} L_{\Sigma}=\Sigma$.

Proof. That $L_{\Sigma}$ is a compact CL-subobject of $L$ follows from [5, 2.10]. Now since $\Sigma \cup\{1\}$ is closed in $L_{\Sigma}$ and order generates it, we have $\operatorname{Spec} L_{\Sigma} \subset$ PRIME $L_{\Sigma} \subset \Sigma \cup\{1\}[5,2.9]$.

We consider the above case in more detail in $\$ 6$.

3.12. TheOREM. Let $L$ be a distributive continuous lattice. Then $\operatorname{Spec} L$ is a locally quasicompact sober space and $\sigma_{\Sigma}: L \rightarrow O(\operatorname{Spec} L)$ is an isomorphism.

Proof. By 2.7 of [5] Spec $L$ order generates $L$ (since the distributivity of $L$ implies that irreducible elements are prime). Hence $L_{\Sigma}=L$. The theorem now follows from 3.8 and 2.3.

This theorem allows us to represent every distributive continuous lattice in the form $O(X)$ for some locally quasicompact sober space $X$. This generalizes the representation of Gierz and Keimel [2].

We refer the reader to Example 2.25 of [5] for a situation where $L_{\Sigma}$ need not be a CL-subobject even if $\Sigma=\operatorname{Spec} L$.

4. Core-compact spaces. In this section we investigate a converse problem to that studied in \$3: Starting from a space $X$, how do we recognize that $O(X)$ is a continuous lattice?

4.1. Definition. A space $X$ is said to be core-compact if for every open set $U, p \in U$, there exists an open set $V$ with $p \in V \subset U$ such that every open cover of $U$ has finitely many members which cover $V$.

4.2. Proposition. Let $X$ be a topological space. The following statements are equivalent.

(1) $X$ is core-compact;

(2) For every open set $U, p \in U$, there exists an open set $V$ with $p \in V \subset U$ such that every filter which has $V$ as a member has a cluster point in $U$;

(3) $O(X)$, the lattice of open sets, is a continuous lattice.

(4) For every open set $U, p \in U$, there exists a Scott-open set $H \subset O(X)$ such that $U \in H$ and $\cap_{V \in H} V$ is a neighborhood of $p$ in $X$.

Proof. (1) $\Leftrightarrow(2)$. It is straightforward to show that for $V \subset U$, every open cover of $U$ has finitely many elements which cover $V$ if and only if every filter which has $V$ as a member has a cluster point in $U$.

(1) $\Leftrightarrow(3)$. For open sets $V$ and $U$ with $V \subset U$, every open cover of $U$ has finitely many elements which cover $V$ if and only if $V \ll U$ in the lattice $O(X)$. This equivalence now follows from the definition of a continuous lattice.

(3) $\Rightarrow(4)$. Let $U$ be an open set in $X, p \in U$. Since $O(X)$ is continuous, there exists $V \in O(X)$ such that $p \in V, V \ll U$. Then $H=\{W \in O(X)$ : 
$V \ll W\}$ is a Scott-open set in $O(X)$ containing $U$. Then we have $p \in V \subset$ $\cap_{W \in H} W$.

(4) $\Rightarrow(1)$. Let $U$ be an open set in $X, p \in U$. Then there exists a Scott-open set $H \subset O(X)$ such that $\cap_{W \in H} W$ is a neighborhood of $p$ and $U \in H$. Let $V \in O(X)$ such that $p \in V \subset \cap_{W \in H} W$. Let $U$ be an open cover of $U$. Since $\uparrow H=H, \cup \mathcal{U} \in H$. Again since $H$ is Scott-open, there exists a finite $\mathscr{F} \subset \mathcal{Q}$ such that $\cup \mathscr{F} \in H$. Thus $V \subset \cup \mathscr{F}$.

Spaces satisfying the equivalent conditions of 4.2 have been investigated in the literature. They are called semilocally bounded by Isbell [9], quasi-locally compact by A. S. Ward [11], and spaces satisfying condition (C) by Day and Kelly [1] (where their condition (C) is precisely equivalence (4) in 4.2).

Let $L$ be a continuous lattice endowed with the Scott topology. Isbell showed that the set of continuous functions from $X$ to $L, L^{X}$, is a continuous lattice with respect to the pointwise order iff $X$ is core-compact [8]. Day and Kelly showed that $X$ satisfies (4) of 4.2 iff $f \times 1_{X}: Y \times X \rightarrow Z \times X$ is a quotient mapping for all quotient maps $f: Y \rightarrow Z$. Their results give important additional equivalences in order that a space be core-compact.

We point out that condition (2) of Theorem 3.8 of the preceding section is by 4.2 the condition that $\Sigma$ be core-compact.

4.3. Definition. If $j: X \rightarrow Y$ is an embedding of topological spaces, then we call $j$ strict if $U \rightarrow j^{-1}(U): O(Y) \rightarrow O(X)$ is an isomorphism of lattices. Observe that a strict embedding is always dense.

Note that for $T_{0}$-spaces $X$, the sobrification mapping $\xi: X \rightarrow \check{X}$ is a strict embedding by 2.7 .

4.4. Lemma. Let $L$ be a distributive continuous lattice, and $X \subset \operatorname{Spec} L$. Then the following statements are equivalent:

(1) The inclusion $X \rightarrow \operatorname{Spec} L$ is a strict embedding (relative to the hullkernel topology on $X$ );

(2) $X$ is order generating in $L$.

REMARK. In $[5,2.2]$ one finds alternative equivalent conditions for condition (2).

Proof. Condition (1) means that for all $s, t \in L$, the relation $\sigma(s) \cap X=$ $\sigma(t) \cap X$ implies $s=t$. This is equivalent to

(1') For all $s, t \in L$, the relation $\uparrow s \cap X=\uparrow t \cap X$ implies $s=t$.

Since $\uparrow s \cap X=\uparrow t \cap X$ is equivalent to $\uparrow s \cap(X \cup\{1\})=\uparrow t \cap(X \cup$ $\{1\})$ we note that $[5,2.2]$ shows that $\left(1^{\prime}\right)$ and (2) are equivalent.

4.5. THEOREM. For a $T_{0}$-space $X$ the following statements are equivalent:

(1) $X$ is core-compact (i.e. $O(X)$ is a continuous lattice);

(2) [resp. (2')] $X$ allows a strict embedding into a locally quasicompact [sober] space; 
(3) There is a continuous distributive lattice $L$ such that $X$ is homeomorphic to a subspace $Y$ of Spec $L$ for which $Y$ is order generating in $L$.

(4) The soberfication $\check{X}$ of $X$ is locally quasicompact.

Proof. (3) $\Rightarrow\left(2^{\prime}\right)$. By $3.12 \mathrm{Spec} L$ is a locally quasicompact sober space. Thus (3) implies (2') by Lemma 4.4 .

$\left(2^{\prime}\right) \Rightarrow(2)$ is trivial.

(2) $\Rightarrow$ (1) follows from 3.6(b) and Definition 4.3.

(1) $\Rightarrow$ (4) follows from 3.12 since $\check{X}=\operatorname{Spec}(O(X))$.

(4) $\Rightarrow(3)$. Let $L=O(X)$. Then $\xi: X \rightarrow \operatorname{Spec} L=\check{X}$ is a strict embedding by 2.7(ii) and Definition 4.3. Thus $\xi(X)$ is order-generating by Lemma 4.4. Also since $O(X)$ and $O(\operatorname{Spec} L)$ are isomorphic, $O(X)$ is continuous by 3.6.

4.6. Corollary. Let $X$ be a sober space. Then the following conditions are equivalent:

(1) $O(X)$ is a continuous lattice.

(2) $X$ is locally quasicompact. Moreover, if these conditions are satisfied, then $U \ll V$ in $O(X)$ iff there is a quasicompact $Q \subset X$ with $U \subset Q \subset V$.

Proof. The equivalence of (1) and (2) follows from the equivalence of (1) and (4) in 4.5. The last statement is a result of 3.6.

4.7. Corollary [1], [9]. For a Hausdorff space $X$ the lattice $O(X)$ is continuous iff $X$ is locally compact.

Theorem 4.5 characterizes $T_{0}$-spaces $X$ for which $O(X)$ is continuous provided one understands the concept of strict dense subspaces of locally quasicompact sober spaces or, alternatively, order generating subsets of PRIME $L$ for distributive continuous lattices $L$. As far as sober spaces are concerned, the core-compact ones are in bijective correspondence with distributive continuous lattices by 3.12 and 4.6 , and are precisely the locally quasicompact ones. In $\$ 7$ we construct an example of a core-compact space $X$ which is not locally quasicompact.

5. The spectra of algebraic lattices. In this section we apply the developments of the preceding sections to algebraic lattices, i.e., objects of $\underline{Z}$. The first theorem is an analog of Theorem 3.8.

5.1. TheOREM. Let $L$ be a complete lattice, $\Sigma \subset \operatorname{Spec} L$. The following statements are equivalent:

(1) $L_{\Sigma}$ is an algebraic lattice;

(2) $\Sigma$ is a $T_{0}$-space with a basis of quasicompact open sets;

(3) Spec $L_{\Sigma}$ is a sober space with a basis of quasicompact open sets. 
Proof. (1) $\Rightarrow(2)$. Let $p \in \sigma_{\Sigma}(x) \subset \Sigma$. Since $L_{\Sigma}$ is algebraic, there exists a compact element $k \in L_{\Sigma}$ such that $k \leqslant x$ but $k \nless p$. Since $k$ is a compact element, $\uparrow k$ is a Scott-open principal filter. Since $\Sigma$ order generates $L_{\Sigma}$, (Irr $\left.L_{\Sigma}\right) \backslash\{1\} \subset \Sigma[5,2.5]$. Thus by 3.3(B) $\uparrow k$ is $\Sigma$-compatible in $L_{\Sigma}$. Hence by $3.4 \sigma_{\Sigma}(\uparrow k)=\sigma_{\Sigma}(k)$ is quasicompact. Also we have $p \in \sigma_{\Sigma}(k) \subset \sigma_{\Sigma}(x)$.

$(2) \Rightarrow(1)$. Note that a quasicompact open set is a compact element of the lattice $O(\Sigma)$. Since the hypothesis every open set is a union of quasicompact open sets, $O(\Sigma)$ is an algebraic lattice. By $2.5 L_{\Sigma}$ is isomorphic to $O(\Sigma)$.

(1) $\Rightarrow$ (3). Note that $L_{\Sigma}$ is order generated by both $\Sigma$ and Spec $L_{\Sigma}$ (since $\operatorname{Spec} L_{\Sigma} \supset \Sigma$ ). Taking $\Sigma=\operatorname{Spec} L_{\Sigma}$, Spec $L_{\Sigma}$ has a basis of quasicompact open sets by the equivalence of (1) and (2). By Theorem 3.8 Spec $L_{\Sigma}$ is sober. (3) $\Rightarrow(1)$ is a special case of $(2) \Rightarrow(1)$ where $\Sigma=\operatorname{Spec} L_{\Sigma}$.

Note that in contrast to the more general case of $L_{\Sigma}$ being a continuous lattice, we have always that $\Sigma$ is locally quasicompact if $L_{\Sigma}$ is an algebraic lattice.

5.2. Corollary. Let $L$ be a distributive algebraic lattice. Then every strictly embedded subspace $\Sigma \subset \operatorname{Spec} L$ is a $T_{0}$-space with a basis of quasicompact open sets, and $\sigma_{\Sigma}: L \rightarrow O(\Sigma)$ is an isomorphism.

We turn now to the characterization of those spaces $X$ for which $O(X)$ is an algebraic lattice (cf. 4.5).

5.3. THEOREM. For $a T_{0}$-space $X$ the following statements are equivalent:

(1) $O(X)$ is an algebraic lattice.

(2) $X$ has a basis of quasicompact open sets.

(3) $X$ admits a strict embedding into a sober space with a basis of quasicompact open sets.

(4) There is a distributive algebraic lattice $L$ such that $X$ is homeomorphic to a subspace $Y$ of $\operatorname{Spec} L$ with $\operatorname{Irr} L \backslash\{1\} \subset Y$.

(5) The sobrification $\check{X}$ of $X$ has a basis of quasicompact open sets.

Proof. By $2.7 \xi: X \rightarrow \operatorname{Spec} O(X)$ is a strict embedding and $\xi(X)$ is homeomorphic to $X$ via $\xi$. Let $\Sigma=\xi(X)$. By Lemma $4.4 \xi(X)$ order generates $O(X)$. Thus $O(X)_{\Sigma}=O(X)$. The equivalence of (1), (2), and (5) then follows from Theorem 5.1.

(5) $\Rightarrow(3)$. Immediate.

(3) $\Rightarrow(1)$. Suppose $j: X \rightarrow Y$ is a strict embedding where $Y$ has a basis of quasicompact open sets. By the equivalence of (1) and (2), $O(Y)$ is an algebraic lattice. Since $O(X)$ is isomorphic to $O(Y), O(X)$ is algebraic.

(4) $\Rightarrow$ (2). Since Irr $L \backslash\{1\} \subset Y, Y$ order generates $L[5,2.5]$. By Lemma 4.4 $Y$ is strictly embedded in $\operatorname{Spec} L$. Thus $Y$ has a basis of quasicompact open sets by 5.2 . 
(1) $\Rightarrow(4)$. Let $L=O(X)$ and consider $\xi: X \rightarrow \operatorname{Spec} O(X)$. As above $\xi$ : $X \rightarrow \xi(X)$ is a homeomorphism and $\xi$ is a strict embedding. By Lemma 4.4 $\xi(X)$ is order generating and hence (Irr $L) \backslash\{1\} \subset \xi(X)$ by [5, 2.5].

6. The patch topology. The patch topology is extensively used in the spectral theory of commutative rings (see e.g. [3]). Here we study it in the context of continuous lattices.

6.1. Definition. Let $X$ be a topological space. For $x, y \in X$ we write $x<y$ if $y \in\{x\}^{-}$. This is a transitive relation and a partial order if $X$ is $T_{0}$. The set $\downarrow Y$ (with respect to this order) is called the saturation of $Y$ for $Y \subset X$, and $Y$ is saturated if $Y=\downarrow Y$.

Note. If $L$ is a complete lattice, then the partial order induced by that of $L$ on $\Sigma \subset$ Spec $L$ agrees with the one given on $\Sigma$ by 6.1.

The following observations are straightforward.

6.2. REMARK. All open sets of a space are saturated. The saturation of a set $Y$ is the intersection of all open sets containing $Y$. The set $Y$ is saturated iff $Y$ is an intersection of open sets. The saturation of a quasicompact set is quasicompact. A space is locally quasicompact iff every point has arbitrarily small saturated quasicompact neighborhoods.

6.3. Proposition. Let $L$ be a complete lattice, $\Sigma \subset \operatorname{Spec} L$. Then $Q \subset \Sigma$ is saturated and quasicompact iff there exists a $\Sigma$-compatible Scott-open filter $F$ in $L$ such that $Q=\Sigma \backslash F$. The function $\sigma_{\Sigma}:(\Theta \mathscr{F}, \cap) \rightarrow(2 \mathcal{S}, \cup)$ from the $n$-semilattice of $\Sigma$-compatible open filters of $L$ into the $U$-semilattice of quasicompact saturated sets in $\Sigma$ defined by $\sigma_{\Sigma}(F)=\Sigma \backslash F$ is an isomorphism. In particular if $L$ is a distributive lattice, and $\Sigma=\operatorname{Spec} L$, then the isomorphism $\sigma_{\Sigma}$ has domain all open filters of $L$.

Proof. Since by earlier remarks the partial order on $\Sigma$ induced by the hull-kernel topology agrees with that induced by $L, Q$ is saturated means $\downarrow Q \cap \Sigma=Q$. The first assertion then follows from Lemma 3.4.

The first assertion implies that the image of $\sigma_{\Sigma}$ is exactly the set of all quasicompact saturated sets. Since $\sigma_{\Sigma}$ clearly reverses order, it remains to verify that $\sigma_{\Sigma}$ is injective. Suppose $F$ and $G$ are $\Sigma$-compatible open filters, $F \neq G$. Then there exists $x \in F \backslash G$ (or vice-versa). Since $G$ is $\Sigma$-compatible, there exists $p \in \Sigma \backslash G$ such that $x<p$. Thus $p \in \Sigma \backslash G=\sigma_{\Sigma}(G)$, but $p \notin \Sigma$ $\backslash F=\sigma_{\Sigma}(F)$.

The final assertion follows from 3.3.

We turn now to a purely topological concept.

6.4. Definition. Let $X$ be a topological space and 1 an element with $1 \notin X$. The patch topology on $Y=X \cup\{1\}$ is the topology generated by $O(X)$ and the collection of all $Y \backslash Q$ where $Q$ is a quasicompact saturated subset of $X$. 
6.5. Remark. If $X$ is $T_{0}$ and locally quasicompact, then $Y$ is Hausdorff. (It is easy to separate $p$ and 1 . If $p \neq q$, there exists $U$, an open set, such that $p \in U, q \notin U$ or vice-versa. Pick a quasicompact neighborhood $Q$ of $p$ such that $Q \subset U$. Then the saturation $P$ of $Q$ is quasicompact and contained in $U$. Then int $Q$ and $Y \backslash P$ separate $p$ and $q$.)

6.6. Proposition. Let $L$ be a continuous lattice, $\Sigma \subset \operatorname{Spec} L$. Then the patch topology on $\Sigma \cup\{1\}$ is coarser than or equal to the topology induced by the CL-topology. If $\Sigma$ is locally quasicompact and $L_{\Sigma}$ is a compact CL-subobject, the two topologies agree (e.g. they agree if $L$ is distributive and $\Sigma=\operatorname{Spec} L$ ).

Proof. Suppose $U \in O(\Sigma)$. Then $U=\sigma_{\Sigma}(x)$ for some $x \in L$. Then $U=\Sigma$ $\cap(L \backslash \uparrow x)$, an open set in the relative CL-topology. Let $V$ be the complement in $\Sigma \cup\{1\}$ of a quasicompact saturated set $Q \subset \Sigma$. By 3.4 we have $Q=\downarrow Q$ $\cap \operatorname{Spec} L$ where $\downarrow Q$ is Scott-closed and hence CL-closed (see e.g. [10, Theorem 13]). Thus $Q$ is closed in the relative CL-topology. Hence $V$ is open in the relative CL-topology. Thus the first assertion.

Now suppose $\Sigma$ is locally quasicompact. By the proposition of $\S 1$ a subbasis for the open sets of $L$ in the CL-topology is given by all sets of the form $\{s \in L: x \nless s\}$ and $\{s \in L: x \ll s\}, x \in L$. But $\{s \in L: x \nless s\} \cap(\Sigma$ $\cup\{1\})=\sigma_{\Sigma}(x)$ is open in the hull-kernel, and hence patch topology. If $p \in(\Sigma \cup\{1\}) \cap\{s \in L: x \ll s\}$, then $x \ll p$. By 3.4 and 3.10 there exists an open filter $F$ such that $p \in F \subset \uparrow x$ and $\Sigma \backslash F$ is quasicompact. Since $F=\uparrow F$, $\Sigma \backslash F$ is saturated. Thus $F \cap(\Sigma \cup\{1\})$ is open in the patch topology and a subset of $\{s \in L: x \ll s\}$ (since it is known that $\{s \in L: x \ll s\}=\operatorname{int}(\uparrow x)$ ).

6.7. TheOREM. Let $L$ be a distributive continuous lattice and $X=\operatorname{Spec} L$. (Note that $X$ is a locally quasicompact sober space and that every such space occurs precisely in this fashion.) Then the following statements are equivalent:

((0)) (Keimel-Mislove) For all $x, a, b \in L$, the relations $x \ll a$ and $x \ll b$ imply $x \ll a b$.

(1) (Keimel-Mislove) PRIME $L$ is closed in $L$.

(2) The collection of saturated quasicompact sets in $X$ is closed under (finite) intersections.

(3) The patch topology on $X \cup\{1\}$ is compact.

If further $L \in \underline{Z}$, i.e., $L$ is an algebraic lattice, then (1)-(3) are equivalent to

(4) (Keimel-Mislove) $L$ is an arithmetic lattice (i.e. $K(L)$ is a sublattice of $L)$.

Proof. $((0)) \Rightarrow(1)$. Suppose $q \notin$ PRIME $L$. Then there exist $a, b \in L$ such that $a \nless q, b \nless q$, but $a b<q$. Pick $c \ll a$ and $d \ll b$ such that $c \nless q$ and $d k q$. Then $c d \ll a$ and $c d \ll b$ imply by hypothesis that $c d \ll a b$. Let $U=\{x: c d \ll x, c k x, d \nless x\}$. By the proposition of $\S 1, U$ is an open set. 
Since $c d \ll a b \leqslant q$, we have $q \in U$. But $U \cap$ PRIME $L=\varnothing$ since $c d \leqslant y$ for all $y \in U$, but $c \nless y$ and $d \nless y$. Thus the complement of PRIME $L$ is open.

(1) $\Rightarrow(2)$. Let $Q$ be a saturated subset of Spec $L$. Then since PRIME $L$ is closed in the compact Hausdorff space $L, Q$ closed in $L$ implies $Q$ is compact. Thus $\downarrow Q=Q L$ is compact and hence closed. By $3.4 Q$ is quasicompact. Conversely if $Q$ is quasicompact by $3.4 \downarrow Q$ is closed. Hence $Q=\downarrow Q \cap$ PRIME $L$ is closed. Thus $Q$ is quasicompact if and only if $Q$ is closed in $L$. Since any collection of closed saturated sets has closed saturated intersection, the implication follows.

$(2) \Rightarrow((0))$. Let $x \ll a, b$. Then $\sigma(x) \ll \sigma(a), \sigma(b)$ by 3.12. By Proposition 3.6 there are quasicompact saturated subsets $P, Q$ in $X$ with $\sigma(x) \subset P \subset \sigma(a)$ and $\sigma(x) \subset Q \subset \sigma(b)$. By (2) $P \cap Q$ is quasicompact, and $\sigma(x) \subset P \cap Q \subset$ $\sigma(a) \cap \sigma(b)=\sigma(a b)$. Thus $x \ll a b$ by 3.6 and 3.12.

(1) $\Leftrightarrow(3)$. Proposition 6.6.

$((0)) \Leftrightarrow(4)$. Let $L$ be an algebraic lattice. Suppose $L$ is arithmetic and $x \ll a, b$. Then there exist compact elements $k, l$ such that $x \leqslant k \leqslant a$ and $x<l<b$. Then $x \leqslant k l \leqslant a b$. Since $k l$ is compact, we have $x<k l \ll k l \leqslant$ $a b$. Thus $x \ll a b$. Conversely if $L$ satisfies $((0))$, let $a$ and $b$ be compact elements of $L$. Then $a b \leqslant a \ll a$ and $a b \leqslant b \ll b$. Hence by ((0)) $a b \ll a b$. Thus $a b$ is compact, and hence $K(L)$ is a lattice.

Note. The equivalence of $((0))$ and $(4)$ and the implications $((0)) \Rightarrow(1) \Rightarrow(2)$ hold without the hypothesis of distributivity (with the same proofs).

The equivalence of $((0))$, (1), and (4) appeared in a Seminar on Continuous Lattices (SCS) memo dated 9-30-76 by Keimel and Mislove. The equivalence of (1) and (4) appeared in [12] and in another SCS memo by Hofmann and Wyler.

6.8. Corollary. Let $L$ be a continuous lattice. If $L$ satisfies condition $((0))$ (or if $L$ is an arithmetic lattice), then $\operatorname{Spec} L \cup\{1\}$ is closed in $L$.

Proof. As remarked earlier the equivalence of $((0))$ and $(4)$ and the implication $((0)) \Rightarrow(1)$ hold in 6.7 even if $L$ is not distributive.

Hence either of the conditions of the corollary imply the case discussed in the latter part of $\S 3$ for $\Sigma=\operatorname{Spec} L$.

6.9. Proposition (Gierz-Keimel [2]). Let $L$ be a distributive continuous lattice in which the equivalent conditions of Theorem 6.7 are satisfied. Then $L$ is isomorphic to the lattice of open decreasing sets in the patch topology of Spec $L$.

The idea here is that $\sigma_{\Sigma}: L \rightarrow O(\operatorname{Spec} L)$ is an isomorphism onto the proper decreasing subsets of Spec $L$. The only work is showing $\sigma_{\Sigma}$ is onto. See [2] for the details. 
We remark that the open decreasing sets of Spec $L$ are anti-isomorphic to the closed nonempty increasing sets in the compact partially ordered space Spec $L \cup\{1\}$. Conversely in a compact partially ordered space with 1 , the closed increasing sets form a continuous lattice with respect to union. Hence distributive continuous lattices $L$ in which PRIME $L$ is closed may be characterized as lattices isomorphic to the set of closed increasing sets in a compact partially ordered space with 1 .

Hochster [3] calls a space spectral if it is quasicompact and sober and if the quasicompact open subsets are closed under finite intersection and form a basis. He proves that a space is spectral if and only if it is homeomorphic to Spec $A$, the space of prime ideals, for a commutative ring $A$ with 1 . It follows easily from 6.7 that a space is spectral if and only if it is homeomorphic to Spec $L$ for an arithmetic lattice $L$ in which 1 is isolated in the set of primes.

Indeed suppose $A$ is a commutative ring with 1 . For each ideal $I$, let $I^{*}$ be the intersection of all prime ideals containing $I$. Define a lattice congruence on the lattice of all ideals of $A$ by $I \sim J$ if $I^{*}=J^{*}$. If $L$ is the quotient lattice, then the prime elements of $L$ are precisely the equivalence classes of the prime ideals in the lattice of ideals, and under this identification $\operatorname{Spec} A$ and $\operatorname{Spec} L$ are homeomorphic.

7. An example. In $\$ 4$ we investigated core-compact spaces which can be defined as spaces $X$ for which $O(X)$ is a continuous lattice. We saw that the following conditions were equivalent: (1) $X$ is core compact, (2) $\check{X}$ is locally quasicompact, and (3) $X$ may be identified with an order generating subset of Spec $L$ for some distributive continuous lattice $L$. The following question remained open (as far as we know first posed by A. S. Ward [11]): Is every core-compact space necessarily locally quasicompact?

Equivalence (3) suggests looking for order generating subsets of Spec $L$ for a counterexample. However the results of $\$ 5$ imply that our search will be vain among algebraic lattices; there all order generating sets have a basis of open quasicompact neighborhoods.

The answer to the question is, however, no. There is in fact a second countable core-compact space in which every quasicompact subspace has empty interior. The lattice $L$ consists of all lower semicontinuous functions from the unit interval $I$ into itself. A function $f: I \rightarrow I$ is lower semicontinuous iff $f$ is continuous when the codomain is endowed with the Scott topology (open sets are of the form $] x, 1]$ ). Hence since $I$ with its usual topology is locally compact and hence core-compact, it follows from Isbell's result [8] that the set of lower semicontinuous functions is a continuous lattice.

Let now $L=\mathrm{LC}(I, I), I=[0,1]$. LC denotes the classically lower semicontinuous functions. For any $(a, b) \in I \times\left[0,1\left[\right.\right.$ let $p_{(a, b)} \in L$ be the lower semicontinuous function given by $p_{(a, b)}(a)=b$ and $=1$ otherwise. We note 
that $L$ is distributive and that $\operatorname{Spec} L=\left\{p_{(a, b)}:(a, b) \in I \times[0,1[\}\right.$. If we equip $Y=[0,1] \times[0,1[$ with the topology consisting of all $\{(x, y) \mid y<f(x)\}$, $f \in \operatorname{LC}(I, I)$, then $(a, b) \mapsto p_{(a, b)}: Y \rightarrow \operatorname{Spec} L$ is a homeomorphism. Notice that $Y$ is second countable.

We define $X \subseteq Y$ as follows. The axiom of choice enables us to fix a subset $A \subseteq I$ with the following properties: (1) $A$ is dense in $I$. (2) $A \cap U$ is not Borel for any open $U \neq \varnothing$ in $I$. (We could have gotten $A$ nowhere Lebesgue measurable in $I$.) We say $(x, y) \in X$ iff $y \in[0,1[$ rational for $x \in A$ and irrational in ]0, 1 [ for $x \in I \backslash A$. If $X^{\prime}$ is the image of $X$ in Spec $L$, then $X^{\prime} \cup\{1\}$ clearly order generates all of Spec $L$ and thus all of $L$. Hence $X$ is core-compact.

In order to show that each quasicompact subset of $X$ has empty interior it suffices to show that every saturated quasicompact subset has empty interior. Thus let $Q$ be a saturated quasicompact subset of $X$. Saturation means that $(a, b) \in Q$ implies $\{a\} \times[0, b] \subseteq Q$.

7.1. Lemma. $q(x)=\max \{y \mid(x, y) \in Q\}$ exists for all $x \in \operatorname{pr}_{1} Q$.

Proof. The collection $Q \cap(\{x\} \times[s-1 / n, s]), n=1,2, \ldots$, where $s=$ $\sup \{y \mid(x, y) \in Q\}$ is a filterbasis of closed subsets of the quasicompact space $Q$ and thus has a nonempty intersection in $Q$. But the only point in this intersection is $(x, s)$.

Define $q: I \rightarrow I$ by $q(x)=0$ for $x \notin \operatorname{pr}_{1} Q$, and as in Lemma 7.1, otherwise.

\subsection{LEMMA. $q: I \rightarrow I$ is upper semicontinuous.}

Proof. Let $x=\lim x_{n}$ in $I$ and suppose that $(x, y)$ is a limit point of $\left(x_{n}, q\left(x_{n}\right)\right)$ in the standard topology of $I \times \mathbf{R}$. By the definition of the topology on $Y$, the relation $(x, y)=\lim \left(x_{n}, q\left(x_{n}\right)\right)$ in the standard topology implies that for any cluster point $(x, z)$ (in $Y)$ of the sequence $\left(x_{n}, q\left(x_{n}\right)\right)$ we have $y \leqslant z$. By the quasicompactness of $Q \cup(I \times\{0\})$, at least one of these cluster points is in $Q \cup(I \times\{0\})$. Thus $y \leqslant q(x)$ by the definition of $q$.

7.3. LEMMA. If $b: I \rightarrow I$ is a Borel function, then $b^{-1}\left(Q^{+}\right)$is a Borel subset of $I$, where $Q^{+}$denotes the set of positive rationals.

Proof. Clear, since $Q^{+}$is Borel.

Now $q$ is a Borel function since it is upper semicontinuous by 7.2 above.

7.4. Lemma. $\left(\operatorname{pr}_{1} Q\right) \cap A$ is a Borel subset of $I$.

Proof. By the definition of $A$ and $X$ we have $\operatorname{pr}_{1} Q \cap A=q^{-1}\left(Q^{+}\right)$.

If $Q$ had a nonempty interior, then $\operatorname{pr}_{1} Q$ would contain a nonempty open subset $U$, whence $A \cap U$ would be a Borel set contrary to the selection of $A$. 
After the construction of this example we found a rather similar construction given by Isbell in [9].

\section{Pseudoprimes.}

8.1. Definition. Let $L$ be a complete lattice. Recall that $I \subset L$ is a prime ideal if $I \neq \varnothing, I=I \vee I=\downarrow I$, and $x y \in I$ implies $x \in I$ or $y \in I$. An element $p \in L$ is called pseudoprime if $p=\sup I$ for some prime ideal $I$. The set of all pseudoprimes is denoted $\psi$ PRIME $L$.

Note the PRIME $L \subset \psi$ PRIME $L$ since $p=\sup \downarrow p$, which is a prime ideal if $p$ is prime.

We recall certain material from [7]. Let $L$ be a continuous lattice. Let PL denote the set of all ideals of $L$. Then PL is a lattice with respect to the operations $I_{1} I_{2}=I_{1} \cap I_{2}$ and $I_{1} \vee I_{2}=\downarrow\left\{a \vee b: a \in I_{1}, b \in I_{2}\right\}$. In fact $\mathrm{PL}$ is an arithmetic lattice in which the compact elements are the principal ideals of $L$, i.e., sets of the form $\downarrow x, x \in L$. The function $r:$ PL $\rightarrow L$ defined by $r(I)=\sup I$ is a CL-morphism. PRIME PL consists of the prime ideals of $L$. Hence $r$ (PRIME PL) $=\psi$ PRIME $L$.

Recall from $[5,1.7]$ that $s \in L$ is a weak prime if $x_{1} \ldots x_{n} \ll s$, then $x_{i} \leqslant s$ for some $i$. The set of all weak primes is denoted WPRIME $L$.

8.2. Proposition. Let $L$ be a distributive continuous lattice. Then $(\operatorname{PRIME~} L)^{-}=$WPRIME $L=\psi$ PRIME $L$.

Proof. The first equality follows from the results of [5].

Suppose $s \in \psi$ PRIME $L$. Then there exists a prime ideal $I$ such that $s=\sup I$. It is shown in [7] that $J=\{y: y \ll s\}$ is the smallest ideal satisfying $\sup J=s$. Hence $J \subset I$. Suppose $x_{1} \ldots x_{n} \ll s$. Then $x_{1} \ldots x_{n} \in$ $J \subset I$. Since $I$ is prime, $x_{i} \in I$ for some $I$. Thus $x_{i} \leqslant s$. Hence $s \in$ WPRIME $L$.

Conversely since PL is arithmetic, PRIME PL is a closed order generating subset (Theorem 6.7 and $[5,3.1]$ ). Hence $r$ (PRIME PL) $=\psi$ PRIME $L$ is a closed order generating subset of $L$. Hence by $[5,2.11](\text { PRIME } L)^{-} \subset$ $\psi$ PRIME $L$.

Note. The fact that $\psi$ PRIME $L$ is closed and that PRIME $L \subset$ $\psi$ PRIME $L \subset$ WPRIME $L$ hold without the hypothesis of distributivity.

8.3. Corollary. Let $L$ be a distributive continuous lattice. Then the various conditions of Theorem 6.7 are equivalent to the condition that if $I$ is a prime ideal of $L$, then $\sup I \in$ PRIME $L$.

Proof. By 8.2 PRIME $L$ is closed iff PRIME $L=\psi$ PRIME $L$ iff for every prime ideal $I$, sup $I \in \operatorname{PRIME~} L$.

The results of this corollary also appear in the previously mentioned seminar report of Keimel and Mislove. 
In general for a distributive continuous lattice $\psi$ PRIME $L$ is a compactification of Spec $L \cup\{1\}$ endowed with the patch (or relative CL-) topology. An interesting question is whether this compactification has some nice categorical characterization, (e.g. is it the "universal" compactification in some category).

In comparing [4] with what is done in this paper one should notice that [4] calls Spec $V$ what we here would have to call Spec PV. We use prime elements as the basic ingredient while [4] uses prime ideals (equivalently, characters). The transition between the two is guaranteed by the functor $P$, which was studied in [7].

In this context we point out how the Stone duality of Boolean algebras and Boolean spaces relates to our results. For a Boolean lattice $L$, the space assigned to it is simply Spec PL. Conversely for a Boolean space $X$, one associates the Boolean lattice of compact elements in the lattice of open sets, $K(O(X))$.

9. Categorical considerations. Let SUP (INF) denote the category with objects complete lattices and morphisms functions which preserve arbitrary sups (infs). By [7] these categories are dual with respect to the functor which is the identity on objects and assigns to a morphism its adjoint (so that the pair form a Galois connection).

For a complete lattice $L$, we define a topology on $L$, called the INF topology, which has as a subbasis of open sets all sets of the form $L \backslash \uparrow x$, $x \in L$. The SUP topology is defined analogously.

9.1. Proposition. Let $f: L \rightarrow M$ be a function between complete lattices satisfying $f(x y)=f(x) f(y)$ for all $x, y \in L$ and $f(1)=1$. Then the following statements are equivalent:

(1) $f$ is continuous for the INF topologies;

(2) $f$ preserves arbitrary infs;

(3) For each $y \in M$, there exists $x \in L$ such that $f^{-1}(\uparrow y)=\uparrow x$.

Proof. (1) $\Leftrightarrow$ (3). Easily (3) implies (1). Conversely for $y \in M, A=f^{-1}(\uparrow y)$ is a subsemilattice of $L$ and $1 \in A$. Let $x=\inf A$. Since $f$ is continuous, $A=A^{-}$. Hence if $x \notin A$, there exist $t_{1}, \ldots, t_{n} \in L$ such that $x \in \cap_{j=1}^{n}(L$ $\left.\backslash \uparrow t_{j}\right)$ and $A \subset \cup_{j=1}^{n} \uparrow t_{j}$. Since $A$ is a subsemilattice $A \subset \uparrow t_{j}$ for some $j$. But then $t_{j} \leqslant x$ since $x=\inf A$, a contradiction. Hence $x \in A$, and thus $f^{-1}(\uparrow y)$ $=\uparrow x$.

(2) $\Leftrightarrow$ (3). Straightforward algebra.

We remark that the INF-SUP duality of [7] may be viewed in the above context as a self-duality on INF. Let $2=\{0,1\}$ be the two element lattice. For a complete lattice $L$, let the dual of $L$ be $\hat{L}=\operatorname{Hom}_{\mathrm{INF}}(L, 2)$. Then by 9.1 the dual corresponds to all principle filters on $L$ (since $f^{-1}(1)$ determines $f$ ). 
This lattice (ordered by inclusion) is anti-isomorphic to $L$, and for $f: L \rightarrow M$, the induced $\hat{f}: \hat{M} \rightarrow \hat{L}$ corresponds (under the natural anti-isomorphisms) to the adjoint $g: M \rightarrow L$.

We note also that for $\Sigma \subset \operatorname{Spec} L$ the hull-kernel topology on $\Sigma$ is just the restriction of the INF-topology on $L$ to $\Sigma$. If $f: L \rightarrow M$ is a SUP-morphism which is an identity preserving lattice morphism, then by 2.4 the left adjoint $g: M \rightarrow L$ carries Spec $M$ into Spec $L$. Since $g$ preserves arbitrary infs, by 9.1 it is INF-continuous, and thus restricted to Spec $M$ is hull-kernel continuous.

Employing the results of $\S \S 2,3$, and 4, we have the following categorical set-up.

9.2. Definition. Let CLSUP denote the category with objects continuous lattices $L$ for which $L_{\mathrm{Spec} L}$, the complete inf-subsemilattice generated by Spec $L$ in $L$, is a CL-subobject and with morphisms SUP-morphisms which also are identity-preserving lattice homomorphisms. Let CTop be the category of all topological spaces $X$ such that $O(X)$ is a continuous lattice and all continuous maps. Let Spec denote the functor from CLSUP to CTop which sends $L$ to $\operatorname{Spec} L$ and $f: L \rightarrow M$ to $\operatorname{Spec} f: \operatorname{Spec} M \rightarrow \operatorname{Spec} L$ (where $\operatorname{Spec} f$ is the restriction and corestriction of the left adjoint $g: M \rightarrow L$ of $f$ ). Let 0 denote the functor from CTop to CLSUP which sends $X$ to the lattice of open sets $O(X)$ and $f: X \rightarrow Y$ to $O(f): O(Y) \rightarrow O(X)$ defined by $O(f)(U)=$ $f^{-1}(U)$.

Recall that a mapping between Hausdorff topological spaces is called proper if the inverse image of compact sets are compact. For non-Hausdorff spaces we modify the definition slightly and say a mapping is proper if the inverse images of saturated quasicompact sets are quasicompact.

9.3. LemMA. Let $f: L \rightarrow M$ in CLSUP. If in addition $f$ is a $\mathrm{CL}^{\mathrm{op}}$-morphism, then $\operatorname{Spec}(f): \operatorname{Spec} M \rightarrow \operatorname{Spec} L$ is proper.

Proof. Let $Q$ be a saturated quasicompact set in Spec $L$. By $3.4 Q=$ Spec $L \backslash F$ for some open filter $F \subset L$. Then $(\operatorname{Spec} f)^{-1} Q=g^{-1}(\operatorname{Spec} L \backslash F)$ $\cap$ Spec $M=\operatorname{Spec} M \backslash g^{-1}(F)$ where $g$ is the left adjoint of $f$. Since $f \in$ $\mathrm{CL}^{\mathrm{op}}$, then $g \in \mathrm{CL}$ and so $g^{-1}(F)$ is an open filter. By 3.10 we know Spec $M \backslash g^{-1}(F)$ is quasicompact.

9.4. Lemma. Let $f: X \rightarrow Y$ in CTop. If $Y$ is sober and $f$ is proper, then $O(F)$ is in $\mathrm{CL}^{\text {op. }}$.

Proof. Let $U \ll V$ in $O(Y)$. Then there is a saturated quasicompact set $Q$ with $U \subseteq Q \subseteq V$ (4.6 and 3.7). Then $O(f)(U) \subseteq f^{-1}(Q) \subseteq O(f)(V)$, and $f^{-1}(Q)$ is quasicompact since $f$ is proper. Then $O(f)(U) \ll O(f)(V)$ by 3.7.

We now add to the umpteen adjunction theorems in [4] another one: 
9.5. Proposition. The assignments Spec: CLSUP $\rightarrow$ CTop and 0: CTop $\rightarrow$ CLSUP are contravariant functors which are adjoint on the right (i.e. Spec: CLSUP $\rightarrow$ CTop $^{\text {op }}$ is left adjoint to 0: CTop ${ }^{\text {op }} \rightarrow$ CLSUP). The adjunctions are $\sigma_{L}: L \rightarrow O(\operatorname{Spec} L)$ and $\xi_{X}: X \rightarrow \operatorname{Spec} O(X)$. The adjunction $\sigma_{L}$ is an isomorphism iff $L$ is distributive and the adjunction $\xi_{X}$ is a homeomorphism iff $X$ is sober locally quasicompact. The functor $0 \circ$ Spec: CLSUP $\rightarrow$ CLSUP is an epireflector onto the full subcategory of distributive continuous lattices, and the functor Spec $\circ$ 0: CTop $\rightarrow$ CTop is an epireflector onto the full subcategory of sober locally quasicompact spaces.

Proof. The adjunction follows from THE FIFTH ADJUNCTION THEOREM 4.3 of $[4$, p. 39] and may also be verified directly. The assertions on the adjunctions come from 2.3 and 2.7 in conjunction with 4.6. The remainder is standard general nonsense.

9.6. THEOREM. The category DCLSUP of distributive continuous lattices with lattice homomorphisms preserving arbitrary sups and the category LQCS of locally quasicompact sober spaces and continuous maps are dual under Spec and 0. Under this duality, the subcategory DCLSUP $\cap \mathrm{CL}^{\mathrm{op}}$ corresponds to the subcategory $\mathrm{LQCP}_{\text {prop }}$ of locally quasicompact sober spaces and proper continuous maps.

This theorem is contained in the FIRST DUALITY THEOREM 4.17 on $p$. 46 of [4]. It adds another case to the SECOND DUALITY THEOREM 5.6 on p. 50 of [4], and this case generalizes the duality between $C_{2}=\underline{Z}$ and the category $K_{2}$ (= full subcategory of LQCP of spaces having a basis of quasicompact open sets). See also Proposition 1.4 on p. 73 of [6].

\section{REFERENCES}

1. B. J. Day and G. M. Kelly, On topological quotient maps preserved by pull-backs or products, Proc. Cambridge Philos. Soc. 67 (1970), 553-558.

2. G. Gierz and K. Keimel, A lemma on primes appearing in algebra and analysis, Houston $J$. Math. 3 (1977), 207-224.

3. M. Hochster, Prime ideal structure in commutative rings, Trans. Amer. Math. Soc. 142 (1969), 43-60.

4. K. H. Hofmann and K. Keimel, A general character theory for partially ordered sets and lattices, Mem. Amer. Math. Soc. No. 122 (1972).

5. K. H. Hofmann and J. D. Lawson, Irreducibility and generation in continuous lattices, Semigroup Forum 13 (1977), 307-353.

6. K. H. Hofmann, M. Mislove and A. Stralka, The Pontryagin duality of compact 0dimensional semilattices and its applications, Lecture Notes in Math., vol. 396, Springer-Verlag, Berlin and New York, 1974.

7. K. H. Hofmann and A. Stralka, The algebraic theory of compact Lawson semilattices-applications of Galois connections to compact semilattices, Dissertationes Math. 137 (1976), 1-58.

8. J. R. Isbell, Function spaces and adjoints, Math. Scand. 36 (1975), 317-339.

9. __ Meet continuous lattices, Sympos. Math. 16 (1975), 41-54. 
10. J. D. Lawson, Intrinsic topologies in topological lattices and semilattices, Pacific J. Math. 44 (1973), 593-602.

11. A. S. Ward, Problem in "Topology and its applications", (Proceedings Herceg, Nov., 1968), Belgrade, 1969, p. 352.

12. O. Wyler, Algebraic theories of contimuous lattices (to appear).

Department of Mathematics, TUlane University, New Orleans, Louisiana 70118

Department of Mathematics, louisiana State University, Baton Rouge, Louisiana 70803 